\title{
Competência para prestar cuidado de enfermagem transcultural à pessoa com deficiência: instrumento de autoavaliação
}

\author{
Competency to provide cross-cultural nursing care for people with disability: a self-assessment instrument \\ Competencia para prestar cuidado de enfermería transcultural a personas con deficiencia: instrumento de \\ autoevaluación
}

\author{
Lorita Marlena Freitag Pagliuca', Evanira Rodrigues Maia" \\ 'Universidade Federal do Ceará, Departamento de Enfermagem. Programa de Pós-Graduação em Enfermagem. \\ Pesquisadora do CNPq. Fortaleza-CE, Brasil. \\ "Universidade Federal do Ceará, Departamento de Enfermagem. Programa de Pós-Graduação em Enfermagem \\ (Doutoranda). Fortaleza-CE, Brasil.
}

\section{Submissão: 22-08-2011 Aprovação: 22-10-2012 \\ RESUMO}

O estudo trata da Teoria de Enfermagem Transcultural que desenvolve fundamentação para o cuidado, essência do trabalho da Enfermagem, apoiada na antropologia que dá suporte e explica aspectos da cultura e do cuidado. Estudo reflexivo apoiado no método de Análise da Teoria para estudo dos conceitos com objetivo de construir Instrumento de Autoavaliação de Competências para o Cuidado Transcultural à Pessoa com Deficiência. Analisados os conceitos principais, Cuidado Culturalmente Competente e Comunicação Cultural, e subconceitos: avaliação, valores, diversidade biocultural, habilidade, conhecimento, identidade, código e empatia cultura. Sintetizou-se o ciclo de análise de valores culturais que subsidiam a autoavaliação. Construiu-se o Instrumento de Autoavaliação da Competência para o Cuidado de Enfermagem Transcultural à Pessoa com Deficiência, especificamente, surdez, cegueira ou baixa visão, deficiência física e mental nos aspectos de cumprimentar, aceitar, ajudar, conhecer e advogar. Conclui-se que a teoria reúne características para o cuidado da pessoa com deficiência.

Descritores: Teoria de Enfermagem; Enfermagem Transcultural; Antropologia; Competência Cultural; Pessoas com Deficiência.

\section{ABSTRACT}

This study addresses the Cross-Cultural Nursing Theory, which develops foundations for care delivery, as the essence of nursing work, based on anthropology, which supports and explains culture and care aspects. This reflexive study was based on the Theory Analysis method to study the concepts aimed at constructing a Self-Assessment Instrument of Competencies for Cross-Cultural Care to Disabled People. After analyzing the main concepts, Culturally Competent Care and Cultural Communication, were analyzed, as well as the sub-concepts: assessment, values, bio-cultural diversity, skill, knowledge, identity, code and cultural empathy. The analysis cycle of cultural values supporting self-assessment was summarized. The Self-Assessment Instrument of Competencies for Cross-Cultural Nursing Care to Disabled People was constructed, specifically deafness, blindness or low sight, physical impairment and mental impairment, regarding greeting, accepting, helping, knowing and advocating. It is concluded that the theory joins characteristics for care delivery to disabled people.

Key words: Nursing Theory; Transcultural Nursing; Anthropology; Cultural Competency; Disabled Persons.

\section{RESUMEM}

El estudio trata de la Teoría de Enfermería Transcultural, que desarrolla fundamentación para cuidar, esencia del trabajo de enfermería, apoyada en la antropología, que soporta y explica aspectos de la cultura y del cuidado. Estudio reflexivo apoyado en el método de Análisis de la Teoría para el estudio de los conceptos, con objeto de construir un Instrumento de Autoevaluación de Competencias para el Cuidado Transcultural a Personas con Deficiencia. Analizados los principales conceptos, Cuidado Culturalmente Competente y Comunicación Cultural, y los subconceptos: evaluación, valores, diversidad biocultural, habilidad, conocimiento, identidad, código y empatía cultural. Se sintetizó el ciclo de análisis de valores culturales que soportan la autoevaluación. Fue construido el Instrumento de Autoevaluación de Competencias para el Cuidado de Enfermería Transcultural a Personas con Deficiencia, específicamente sordera, ceguera y baja visión, deficiencia física y deficiencia mental, respecto a los aspectos de cumplimentar, aceptar, ayudar, conocer y abogar. Se concluye que la teoría reúne características para el cuidado a la persona con deficiencia.

Palabras clave: Teoría de Enfermería; Enfermería Transcultural; Antropología; Competencia Cultural; Personas con Discapacidad.

Projeto financiado pelo Conselho Nacional de Desenvolvimento Científico e Tecnológico (CNPq), com Bolsa de Doutorado do Programa de Pós-Graduação em Enfermagem da Universidade Federal do Ceará (UFC).

\section{AUTOR CORRESPONDENTE～Lorita Marlena Freitag Pagliuca E-mail: pagliuca@ufc.br}




\section{INTRODUÇÃO}

Na Enfermagem, a essência do trabalho é o cuidado, que deve ser amparado por um conjunto de conhecimentos construídos no intuito de produzir um caminho para pensar o propósito, a expressão e o processo para o seu desenvolvimento. Ao longo do último século, enfermeiros produziram teorias com vistas a embasar cientificamente os processos de ensino, de pesquisa e das práticas relacionadas ao trabalho de enfermagem.

Teoria pode ser definida como abstração sistemática da realidade, ou estruturação rigorosa e criativa de ideias que projetam uma visão provisória objetiva e sistematizada dos fenômenos. Assume caráter de sistematização especulativa, transitória, relativa, imperfeita e aproximada do observado na realidade ${ }^{(1)}$. A maioria das teorias tem a perspectiva de compreender e explicar os aspectos biopsicossocio-culturais envolvidos no processo saúde/doença/cuidado de indivíduos, famílias, grupos e comunidades aos quais se destina o cuidado de enfermagem. São elementos constitutivos dessas teorias os conceitos de enfermagem, ambiente/sociedade, saúde e pessoa.

Neste estudo, o Modelo de Análise de Teoria adotado consiste na descrição e na análise crítica da teoria de enfermagem observando sua aplicação à pesquisa, à prática e ao ensino de enfermagem. Descrever e refletir são processos diversos. Descrever é um relato sobre a teoria; refletir é questionar a que propósito serve a teoria. Quando se descreve, atenta-se se a teoria possui características identificadas e organizadas a partir de questões relativas ao processo de cuidar. Estas informações são essenciais para se compreender como funciona e como se trabalha com uma teoria ${ }^{(1)}$.

Construído o arcabouço teórico-conceitual, constitui-se interesse da Enfermagem realizar análise crítica das teorias e dos modelos teóricos elaborados pela profissão, com vistas a testar e validar resultados e contribuir para o seu aperfeiçoamento. Como se evidencia, há teorias que seguem um mesmo eixo norteador, entre elas as teorias de enfermagem antropológica ou cultural. Isto porque as diversidades culturais e étnicas repercutem no cuidado, justificando a formulação de modelos teóricos de enfermagem voltados a explicar a relação entre cuidado humano e cultura.

O pensamento antropológico incorpora a interpretação das práticas e dos discursos buscando compreender as diferenças, pois considera as incertezas e as experiências vividas como partes constituintes de um sistema de significado simbólico que alicerça as estruturas conceituais dos fenômenos sociais ${ }^{(2)}$.

Teorias com abordagem cultural é uma especialidade dentro da Enfermagem, pois têm como meta desenvolver um corpo de conhecimentos científico e humanístico que focalize seus estudos na comparação e análise das diferentes culturas, na perspectiva de examinar com atenção e respeito o comportamento dos sujeitos, a fim de prover cuidados de enfermagem baseados em aspectos específicos e universais ${ }^{(3)}$. Assim, uma revisão sobre esses modelos apontou Leininger, Campinha-Bacote, Giger e Davidhizar, Orque Purnel e Paulanka, Spector, e Andrews e Boyle como as principais teóricas a contribuírem para o avanço do conhecimento de enfermagem transcultural. As duas últimas autoras vêm colaborando nesse campo ao construírem a Teoria Enfermagem Transcultural na perspectiva de prover cuidados de enfermagem culturalmente competentes ou correspondentes ${ }^{(4)}$. Mas o termo competência cultural é novo na literatura de enfermagem, e o mesmo carece ser apresentado e analisado.

Competência cultural pode ser definida como um conjunto de comportamentos harmoniosos, atitudes e políticas reunidas na formação de profissionais que os capacita para trabaIhar de modo eficaz em situações interculturais. Desse modo, competência implica a capacidade para funcionar eficazmente como um indivíduo e uma organização dentro do contexto das convicções culturais, comportamentos e das necessidades dos consumidores e suas comunidades ${ }^{(5-7)}$.

A Teoria de Enfermagem Transcultural é complexa. Para aplicá-la o enfermeiro deve ter condições de interagir com pessoas observando os valores culturais do ser cuidado ${ }^{(4)}$. Para isso, necessita se autoavaliar e ajuizar se está apto a assumir a relação de cuidador com o ser cuidado em diferentes contextos e cenários. A autoavaliação do enfermeiro deve considerar conflitos étnicos e raciais; problemas sociais e religiosos; dificuldades físicas e mentais e, políticas inclusivas. Embora a abordagem para inclusão dos indivíduos com deficiência seja citada na Teoria, não oferece um instrumento com conteúdo próprio para esta autoavaliação(3).

No Brasil, estimativas apontam que $14,5 \%$ da população apresenta algum tipo de deficiência, ou seja, 24,6 milhões de pessoas, número significativo e influenciado pelas condições de acesso à saúde ${ }^{(8)}$. Para a Organização Mundial da Saúde, pessoa com deficiência é aquela com algum tipo de alteração motora, sensorial e mental(9). Como referido, a deficiência é um conceito histórico e culturalmente elaborado. Nesse caso, as tipificações, que estabelecem estereótipos às pessoas com deficiência, são estabelecidas pelos grupos sociais e introjetadas para si e para os outros ${ }^{(10)}$.

A inclusão da pessoa com deficiência exige adaptação e apoio das instituições e espaços públicos e privados em face das diferenças e das necessidades individuais dos sujeitos com deficiência ou necessidades especiais, bem como, o desenvolvimento de competências profissionais podem ser considerado o principal limite à prestação do cuidado inclusivo.

Ao propor uma autoavaliação das atitudes e valores dos enfermeiros em face das variações bioculturais dos sujeitos do cuidado e grupos sociais, a Teoria Enfermagem Transcultural ${ }^{(3)}$ tem como pressuposto prover um processo de comunicação adequado. Isto inclui cuidar e comunicar-se adequadamente com aqueles que vivem com deficiência.

Assim, este estudo objetiva descrever os conceitos da Teoria Enfermagem Transcultural, analisar sua estrutura de intervenção e adaptar Instrumento de Autoavaliação da Competência para o Cuidado de Enfermagem Transcultural para a Pessoa com Deficiência.

\section{MÉTODO}

Trata-se de estudo de reflexão crítica na qual se utiliza um Modelo de Análise de Teoria, que afirma que os componentes 
dos conceitos dizem respeito a como a teoria se estrutura a partir de suas expressões pela linguagem. Também oferece subsídio para avaliar a construção de instrumentos sugeridos pelas teorias ${ }^{(1)}$.

Selecionada a teoria de interesse, procedeu-se à leitura seletiva e tradução da Teoria Enfermagem Transcultural(3) a fim de identificar os conceitos abordados e os instrumentos de autoavaliação para a prática profissional do enfermeiro. Em continuidade, fez-se leitura analítica da Teoria com o objetivo de responder às questões propostas pelo Modelo de Análise de Teoria': Existe conceito principal? Quantos conceitos existem? Há subconceitos organizados?

Para finalizar, procedeu-se à análise do Instrumento de Autoavaliação proposto pela Teoria Enfermagem Transcultural e, em seguida, é desenvolvida a adaptação dos referidos instrumentos para o enfermeiro avaliar suas competências para prestar Cuidado Culturalmente Competente à Pessoa com Deficiência.

\section{RESULTADOS E ANÁLISE}

\section{A Teoria Enfermagem Transcultural e seus conceitos}

Margaret M. Andrews e Joyceen S. Boyle são, respectivamente, docentes do Departamento do Nazareth College of Rochester e do Departamento de Enfermagem Comunitária da Medical College of Georgia. Em 1989, publicaram o livro Modelo Conceitual do Cuidado de Enfermagem Transcultural, resultado de tese de doutorado na Universidade de Utah, cujo objetivo foi clarificar a compreensão dos conceitos de enfermagem cultural para a prática clínica. Atualmente na quinta edição, o livro propõe-se a discutir a construção das bases teóricas da prática de enfermagem e facilitar a realização do cuidado culturalmente competente ${ }^{(11)}$.

De acordo com a análise da Teoria de Enfermagem Transcultural $^{(3)}$ à luz do Modelo de Análise de Teoria ${ }^{(1)}$, as teóricas mencionam dois conceitos principais ou maiores: (1) Cuidado Culturalmente Competente e (2) Comunicação Cultural, e, ainda, oito subconceitos ou conceitos menores: avaliação cultural, valores culturais, diversidade biocultural, habilidade cultural, conhecimento cultural, identidade cultural, código cultural e empatia cultural(1-3).

Cuidado Culturalmente Competente, primeiro conceito principal, consiste no conhecimento dos componentes do cuidado de enfermagem cultural. Diante dos conceitos maiores expressos na teoria, competência pode ser definida como um conjunto de saberes e práticas necessárias a uma profissão para o desenvolvimento dos papéis sociais e exercício profissional, combinando habilidades, conhecimentos e atitudes de modo autônomo ${ }^{(3)}$.

Desenvolvimento de competência cultural para o cuidado efetivo de saúde requer do provedor de cuidados ações contínuas no contexto cultural das pessoas e grupos para alcançar habilidade cultural ${ }^{(12)}$. A competência cultural articula temas como consciência cultural: exame da própria cultura e dos preconceitos do cuidador; conhecimento cultural: conhecer as culturas e grupos étnicos; habilidade cultural: conhecimento cultural e aptidão para avaliação física culturalmente fundamentada; encontro cultural: compreender as variações da biocultura; e desejo cultural: envolve a capacidade de importar-se com o outro. Este é o elemento mais complexo no desenvolvimento de competências. O termo competência cultural é intricado, pois ser competente significa ser satisfatório e estar apto a cuidar é promover a satisfação dos usuários conforme aspectos subjetivos intrínsecos à cultura dos recebedores do cuidado.

Prestar cuidado de enfermagem culturalmente competente ou correspondente condiciona à utilização de um modelo conceitual de enfermagem que constrói consciência e habilidade para o desempenho do cuidado. Desse modo, o desenvolvimento de habilidades para prover Cuidado Culturalmente Competente pauta-se na observação e conhecimento da cultura dos grupos sociais.

O segundo conceito maior da Teoria Enfermagem Transcultural é o de Comunicação Cultural, imperativo para entender as influências culturais na relação enfermeiro/paciente e no processo saúde/doença. É descrito como um sistema organizado e padronizado que regula o comportamento e marca as possíveis interações entre o enfermeiro e o paciente. Pode ser entendido como troca de mensagens e criação de significados ${ }^{(3)}$.

Na Comunicação Cultural, o contexto é significativo e engloba o tom de voz empregado, o ambiente onde a ação está ocorrendo e os aspectos inerentes à linguagem dos povos. Assim, a comunicação desponta como elemento capaz de dirimir barreiras culturais, pois comportamentos adotados na saúde/doença dos grupos sociais podem ser traduzidos na comunicação intercultural. Destaca a variação dos padrões de comunicação entre culturas, sendo premente não negligenciar a comunicação não verbal, pois silêncio, olhar, toque têm significado cultural, e são fundamentais à clínica ${ }^{(3)}$.

Inegavelmente, o significado atribuído às doenças e síndromes guarda relação com a cultura, considerada a partir de experiências e vivências dos indivíduos. Nesse atributo, a percepção de saúde/doença correlaciona-se e fundamenta-se conforme normas culturais e aspectos psicossociais. A tradução ou decodificação desses significados confere a possibilidade do Cuidado Cultural Competente ou Correspondente e pressupõe a observação de dois subconceitos: avaliação cultural e valores culturais.

Avaliação cultural consiste na análise sistemática das convicções, valores e práticas dos grupos para selecionar intervenções de enfermagem contextualizadas. Na avaliação cultural aplicam-se aspectos como comunicação verbal e não verbal, afiliação cultural, orientação de valores, restrições e sanções culturais, convicções relativas a práticas alimentares e condições socioeconômicas. Portanto, avaliação cultural é um atributo a ser observado na construção da competência cultural do enfermeiro ${ }^{(4)}$.

Como parte dos seus estudos, as teóricas apresentam o Guia de Avaliação de Enfermagem Transcultural que objetiva identificar como estes elementos estão explicitados na cultura dos sujeitos do cuidado. A avaliação cultural permite conhecer a relação de saúde/doença considerando raça/cor/etnia e as variações bioculturais relativas à incidência e prevalência 
de doenças e agravos entre os grupos. Essa avaliação também inclui a autoavaliação do enfermeiro para prestar cuidados de enfermagem adequados a seus clientes. Já valores culturais são expressões que dão ordem, direção e significado às decisões, caminhos e ações escolhidas por um grupo social, pois variam entre e dentro de uma mesma cultura ${ }^{(3)}$.

Ainda conforme as teóricas, a autoavaliação da competência para o cuidado da Teoria Enfermagem Transcultural obedece a um modelo de análise que orienta o enfermeiro a analisar ciclicamente como se relaciona com os diferentes grupos na sociedade e descobrir os distintos níveis de resposta que poderia ter para uma pessoa acerca de: 1 . Cumprimentar: sinto que posso cumprimentar esta pessoa calorosamente e dar as boas-vindas a ela sinceramente; 2 . Aceitar: sinto que posso aceitar esta pessoa honestamente e de modo confortável e também os seus problemas; 3. Ajudar: sinto que posso ajudar esta pessoa, com seus problemas relacionados e com os estereótipos construídos pelos problemas por ela enfrentados; 4. Conhecer: sinto que detenho conhecimento para cuidar desta pessoa; e 5. Advogar: sinto que posso honestamente defender esta pessoa ${ }^{(3)}$.

As respostas são apenas para o conhecimento pessoal do enfermeiro, portanto, deve-se responder o que realmente se pensa. Estas respostas devem ser dadas com honestidade, pois servem para o seu conhecimento pessoal acerca de como se comportaria diante de pessoas de diferentes culturas ou em situações conflituosas. Apresenta-se, em seguida, a figura síntese desta ideia.

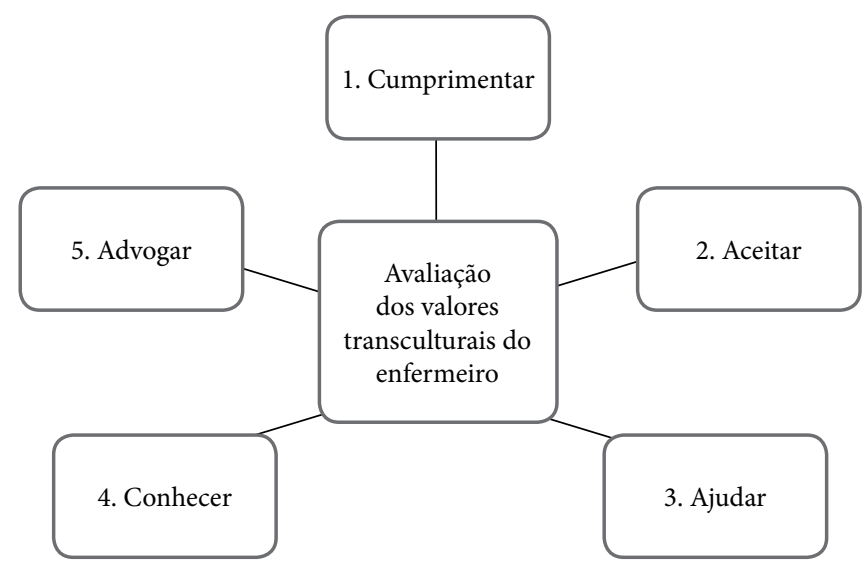

Figura 1 - Ciclo de análise de valores culturais de enfermagem. Construído com base na teoria de Andrews \& Boyde, 2005.

Este ciclo visa organizar a autoavaliação das competências do enfermeiro para prestar Cuidado Culturalmente Competente ou Correspondente. Assim o profissional analisa se cumprimenta, aceita, ajuda, tem convicção de suas habilidades para cuidar e se advogaria em favor de diferentes pessoas. A perspectiva é testar a habilidade do enfermeiro para prover cuidados competentes em situações conflituosas ${ }^{(3)}$.
Há três elementos envolvidos na autoavaliação do enfermeiro: a atitude, a habilidade instrumental e o compromisso social. Quando avalia se está preparado para cumprimentar e aceitar o paciente, demonstra competências de atitude. Quando responde se está apto para ajudar e se conhece a situação biocultural do paciente, evidencia competências para o cuidado instrumental de enfermagem. Advogar ou defender o paciente confirma envolvimento social.

Portanto, para o enfermeiro estar capacitado culturalmente significa que deve cumprimentar e aceitar a pessoa, ajudar e conhecer seus problemas e advogar nos contextos socialmente construídos. No tocante à pessoa com deficiência, deve avaliar se está preparado para prestar cuidado aos deficientes auditivos, visuais, físicos e mentais.

\section{Pressupostos do Instrumento de Autoavaliação da Compe- tência para Cuidado de Enfermagem Transcultural}

Ao compreender que intervenções efetivas de enfermagem sejam possíveis, se baseadas na avaliação das competências de atitudes e aquisição de habilidades clínicas do enfermeiro, utilizar instrumento de autoavaliação é uma estratégia com vistas ao Cuidado Culturalmente Competente ou Correspondente, portanto busca analisar atitudes, valores e convicções que influenciam a prática permitindo formar as habilidades necessárias para o Cuidado de Enfermagem Transcultural.

Assim, é desafio para a Enfermagem prover cuidados em consonância aos valores culturais e o processo saúde/doença dos clientes. Isto porque as diferentes culturas atribuem valores e significados às práticas de saúde, os quais, quando identificados, devem ser correlacionados a expectativa dos sujeitos. Logo, a autoavaliação do enfermeiro pode ser empregada no contexto de saúde/doença daqueles que vivem com necessidades de cuidado especiais, não apenas culturais ou raciais, mas de ordem física, sensorial e mental. Tal situação inclui a assistência a pessoa com deficiência.

De modo geral, a influência da cultura explicita-se tanto nos aspectos comuns como naqueles específicos aos enfermeiros e clientes. Nessa perspectiva, os elementos culturais da profissão influenciam diretamente o processo de cuidar, representado simbolicamente na missão e nos objetivos da profissão, e se materializam nas ações de enfermagem. As teóricas descrevem seu processo de enfermagem pautado em conceitos e subconceitos interrelacionados, amparados por um conceito comum, o de cultura. Este deve subsidiar valores profissionais no processo de cuidado. Mas a não observância desses fatores pode levar a imposição de convicções, valores e padrões culturais próprios ${ }^{(12)}$.

Ainda segundo as teóricas, o instrumento de autoavaliação proposto pela Teoria Enfermagem Transcultural pressupõe antecipar as dificuldades/facilidades do enfermeiro em cuidar de trinta diferentes tipos de indivíduos agrupados em cinco categoriais: étnicos/raciais (americano nativo, negro americano, mexicano americano, anglo-saxão branco, vietnamita americano, haitiano); assuntos/problemas sociais (abusador de criança, prostitutas, gays/lésbicas, alcoólatras, usuários de drogas, adolescente grávida solteira); prejuízos físicos/mentais (pessoa senil ou anciã, pessoa com paralisia cerebral, 
amputados, pessoa com câncer, pessoa com AIDS, pessoa com hemofilia); religiosos (judeus, protestantes, católicos, testemunha de Jeová, harecrisna e ateu) e políticos (membro da ku klux klansman, neonazista, proponente de armamento nuclear, comunista, sindicalista, proponente de união entre pessoas do mesmo sexo). Assim, o ideal é dotar o enfermeiro de conhecimento específico acerca das suas dificuldades/facilidades para prestar cuidado efetivo e inclusivo ${ }^{(3)}$.

Destaque-se, porém, a atenção e as intervenções devem coadunar-se à singularidade e identidade cultural dos sujeitos do cuidado, e o enfermeiro deve avaliar se reúne os atributos necessários para prestar o Cuidado Culturalmente Competente.

Instrumento de Autoavaliação de Competência Transcultural para cuidar da Pessoa com Deficiência

Neste estudo, construiu-se instrumento específico para a autoavaliação do enfermeiro quanto à sua competência cultural para cuidar da pessoa que vive com deficiência. Como exposto, ao responder ao instrumento o enfermeiro deve autoavaliar sua competência para o cuidado assinalando no preenchimento do instrumento se ele se considera culturalmente competente para cada uma destas situações. Com esta finalidade, deve usar as letras $\mathrm{C}$ para competente, $\mathrm{P}$ para parcialmente competente $\mathrm{e}$ I quando se avaliar incompetente para prestar assistência de enfermagem nos componentes do ciclo, variáveis de cumprimentar a advogar pelos sujeitos do cuidado.
Consoante recomendado, o Instrumento de Autoavaliação de Competência Transcultural Para Cuidar da Pessoa com Deficiência deve permitir explorar os processos e os conteúdos para a avaliação cultural inclusiva. Admite-se que a autoavaliação cultural deve aumentar a consciência do enfermeiro diante das atitudes, valores, convicções, e práticas que influenciem sua habilidade para prover cuidado de enfermagem culturalmente competente ou correspondente de indivíduos, famílias, e grupos de culturas diversas no contexto da pessoa com deficiência. De acordo com a Teoria Enfermagem Transcultural, o profissional deve realizar a autoavaliação mediante a identificação de variações bioculturais em saúde e doença para que empreenda um processo de comunicação transcultural como um componente integrante do cuidado de enfermagem transcultural ${ }^{(3)}$.

A aplicação do Instrumento de autoavaliação da competência do enfermeiro para o Cuidado de Enfermagem Transcultural para cuidar da Pessoa com Deficiência deve ser precedida da seguinte explicação ao profissional: Responda honestamente se você se considera culturalmente competente para cada uma destas situações usando as seguintes expressões: Competente - C; Parcialmente competente - P; Incompetente - I. Esta análise sistemática das convicções, valores e práticas do profissional compreende Cumprimentar cordial e sinceramente a pessoa com deficiência; Aceitar a pessoa com deficiência e seus problemas; Ajudar a pessoa com deficiência respeitando seus estereótipos; deter Conhecimento para cuidar da pessoa com deficiência e se Advogar e defender a pessoa com deficiência.

\begin{tabular}{|c|c|c|c|c|c|}
\hline \multirow{2}{*}{ Tipos de Deficiência } & \multicolumn{5}{|c|}{ Níveis de resposta } \\
\hline & Cumprimentar & Aceitar & Ajudar & Conhecer & Advogar \\
\hline \multicolumn{6}{|l|}{ Surdez } \\
\hline \multicolumn{6}{|l|}{ Surdo parcial } \\
\hline \multicolumn{6}{|l|}{ Surdo parcial sem prótese } \\
\hline \multicolumn{6}{|l|}{ Surdo total } \\
\hline \multicolumn{6}{|l|}{ Surdo total com prótese } \\
\hline \multicolumn{6}{|l|}{ Malformação da orelha } \\
\hline \multicolumn{6}{|l|}{ Ausência de pavilhão auricular } \\
\hline \multicolumn{6}{|l|}{ Deformidades ósseas } \\
\hline \multicolumn{6}{|l|}{ Cegueira e baixa visão } \\
\hline \multicolumn{6}{|l|}{ Baixa visão } \\
\hline \multicolumn{6}{|l|}{ Cego parcial } \\
\hline \multicolumn{6}{|l|}{ Cego total sem deformidades } \\
\hline \multicolumn{6}{|l|}{ Cego com deformidades } \\
\hline \multicolumn{6}{|l|}{ Deficiência física } \\
\hline \multicolumn{6}{|l|}{ Cadeirante } \\
\hline \multicolumn{6}{|l|}{ Pessoa com paralisia } \\
\hline \multicolumn{6}{|l|}{ Pessoa com paresia } \\
\hline \multicolumn{6}{|l|}{ Amputado sem prótese } \\
\hline \multicolumn{6}{|l|}{ Amputado com prótese } \\
\hline \multicolumn{6}{|l|}{ Amputações com feridas } \\
\hline \multicolumn{6}{|l|}{ Malformação congênita } \\
\hline \multicolumn{6}{|l|}{ Deficientes físicos acamados } \\
\hline \multicolumn{6}{|l|}{ Deficiência mental } \\
\hline \multicolumn{6}{|l|}{ Déficit de aprendizagem } \\
\hline Déficit no comportamento & & & & & \\
\hline
\end{tabular}

Figura 2 - Instrumento de autoavaliação da competência do enfermeiro para o Cuidado de Enfermagem Transcultural para cuidar da Pessoa com Deficiência. Adaptado com base na teoria de Andrews \& Boyde, 2005. 
Segundo o ciclo de análise de valores culturais, o encontro entre o enfermeiro e a pessoa com deficiência tem início com cumprimentar, isto é, estar aberto, disponível e acolher. Para que o cumprimento seja eficiente e caloroso, os envolvidos devem compartilhar o mesmo código de linguagem verbal e não verbal. Pessoas com limitação sensorial visual e/ou de audição desenvolvem linguagens específicas, códigos táteis ou linguagem de sinais ${ }^{(13)}$. Conforme evidenciado por estudo sobre comunicação verbal e não verbal de mães cegas, a comunicação verbal está preservada entre a mãe e seu filho, mas a resposta não verbal da mãe é pobre tanto para a criança como para o enfermeiro ${ }^{(14)}$. Caso não saiba como superar esta diferença biocultural, o enfermeiro pode sentir-se competente, parcialmente competente ou não competente para o cuidado ao deficiente visual.

Consoante estudo sobre a comunicação de enfermeiros com pacientes surdos em unidades básicas de saúde, esta população é referida como aquela com a qual o enfermeiro menos se sente preparado para manter comunicação e solicita a presença de um familiar do paciente para isto. Cria, assim, outras barreiras culturais, pois, às vezes, o deficiente auditivo quer comentar determinados assuntos apenas com o profissional de saúde, sem a intermediação de outras pessoas ${ }^{(15)}$.

No segundo passo do ciclo de autoavaliação para prestar o cuidado culturalmente competente o enfermeiro deve julgar se aceita a pessoa da qual irá cuidar. Estudo que ouviu deficientes motores, auditivos e visuais apresenta significados sobre crenças e valores, com forte conotação excludente; profissionais da saúde corroboram estes discursos ${ }^{(16)}$. Conforme observado, os currículos da graduação em enfermagem não contemplam plenamente a formação do futuro profissional para cuidar da pessoa com deficiência. Em disciplina optativa específica sobre o tema, alunos foram sensibilizados e relataram estar aptos a estabelecer comunicação introdutória e abordagem segura. Deixaram de perceber a pessoa com deficiência como desvalida e adotaram sentimento de aceitação ${ }^{(17)}$.

Para ajudar a pessoa com deficiência é preciso estar informado dos direitos legais desta pessoa. Neste aspecto, a Política Nacional de Saúde da Pessoa com Deficiência (PNSPD) anuncia seus propósitos: proteger a saúde da pessoa com deficiência; reabilitar a pessoa na sua capacidade funcional e desempenho humano e desta forma contribuir para sua inclusão e prevenir agravos que possam determinar deficiências ${ }^{(18)}$.

Estudo sobre as possibilidades e limites de aplicação da PNSPD aponta a existência de lacunas na escolarização e no acesso ao trabalho da pessoa com deficiência ${ }^{(18)}$. Contudo, como reconhecido na área da saúde, habilitar e reabilitar esta pessoa agrega valor ao cuidado de enfermagem. Portanto, se este cuidado for culturalmente competente tende a ser mais eficaz.

Para cuidar com competência cultural, o enfermeiro deve conhecer o paciente e sua condição biocultural. Por conseguinte, além de cumprimentar com comunicação eficaz, aceitar suas limitações, ter disposição para ajudar, é preciso deter conhecimento técnico-científico sobre a deficiência do paciente e sobre como minimizar-lhe as perdas ${ }^{(19)}$. Para tanto, os subconceitos descritos na Teoria Enfermagem Transcultural, constitui-se competência essencial do enfermeiro realizar exame físico para reconhecer diferenças bioculturais implicadas na deficiência. Esta distinção deve subsidiar um exame físico plural. Observar tais variações requer conhecimento acurado tanto dos padrões de exame físico como dos aspectos específicos das pessoas que vivem com deficiência e suas necessidades de cuidado. Estas pessoas guardam uma diversidade biocultural relativa à sua cultura e sua saúde presentes na atribuição de significados aos sintomas a serem etnografados pelos enfermeiros, os quais devem ser avaliados e considerados no contexto sociocultural e na etnohistória destas pessoas ${ }^{(3)}$.

Ao planejar os cuidados de vida diária de adolescente que desenvolveu deficiência visual decorrente de diabetes, o enfermeiro, ao interagir com a jovem, buscou entender seu estilo de vida anterior e as transformações ocorridas na presente situação ${ }^{(20)}$. Esta conduta incorpora componentes culturais em uma circunstância de fortes mudanças, em que a enfermeira deve dominar habilidades de comunicação e avaliação da saúde biológica e social.

Para advogar pela pessoa com deficiência, o enfermeiro, deve estar atento à teia complexa de fatores e conceitos combinados na prestação de cuidado de enfermagem. Deve-se destacar a premissa de explorar componentes da comunicação, como a empatia cultural, para realizar o cuidado de enfermagem da pessoa com deficiência, voltado para aqueles que vivem com deficiência e não a seus cuidadores.

O uso clínico da Enfermagem Transcultural para o cuidado da pessoa com deficiência consiste na provisão de cuidados de enfermagem culturalmente competentes ou correspondentes. Desta forma, o processo de enfermagem dessas teóricas não trabalha na perspectiva da repadronização cultural, e sim, propõem compreensão da variação biocultural dos aspectos de saúde/doença dos indivíduos e grupos. Nesse caso, a perspectiva é o cuidado inclusivo de enfermagem com respeito à cultura e as diferenças. Assim, o enfermeiro deve avaliar suas competências para cuidar.

Portanto, observa-se a possibilidade de aplicação da Teoria Enfermagem Transcultural ao contexto das pessoas com deficiência, principalmente considerando a amplitude da problemática que afeta significativa parcela da população, em variados cenários culturais. Neste âmbito, as distintas características socioeconômicas, alimentares, de linguagem, composição étnica, manifestações populares, incidência e prevalência de doenças e agravos à saúde, pressupõem distinção na ocorrência das deficiências físicas, mentais e sensoriais acompanhadas de diferentes percepções pelos enfermeiros.

O reconhecimento dessa diversidade biocultural exige a capacitação do enfermeiro para garantir a promoção do Cuidado Culturalmente Competente às populações. Faz-se, necessária a adoção do processo de Cuidado Culturalmente Competente como suporte do processo de cuidar em enfermagem.

\section{CONCLUSÕES}

A essência do trabalho da Enfermagem é o cuidado. Teorias de enfermagem desenvolvem fundamentação para o cuidado e para a prática e a antropologia dá suporte para teorias que explicam as questões da cultura e do cuidado. Este estudo 
de reflexão teve como referencial metodológico um Modelo de Análise de Teoria com a seleção dos conceitos da Teoria Enfermagem Transcultural. Analisaram-se os dois conceitos principais, a saber, (1) Cuidado Culturalmente Competente e (2) Comunicação Cultural, e oito subconceitos discutidos transversalmente aos conceitos principais, a saber: avaliação cultural, valores culturais, diversidade biocultural, habilidade cultural, conhecimento cultural, identidade cultural, código cultural e empatia cultural. Como referido, a Teoria Enfermagem Transcultural descreve o ciclo de análise que subsidia a autoavaliação dos valores do enfermeiro, a saber: cumprimentar, aceitar, ajudar, conhecer e advogar.

A última etapa do estudo foi construir o Instrumento de Autoavaliação da Competência para o Cuidado de Enfermagem
Transcultural para a Pessoa com Deficiência. Este instrumento lista surdez, cegueira e baixa visão, deficiência física e deficiência mental, enumerando diferentes situações. Em seguida são disponibilizados os níveis de respostas do ciclo de análise dos valores transculturais para avaliar a competência do enfermeiro quanto a cumprimentar, aceitar, ajudar, conhecer e advogar em favor da pessoa com deficiência.

Conclui-se este estudo com a apresentação do Instrumento de Autoavaliação da Competência para o Cuidado Transcultural para a Pessoa com Deficiência possibilitando avaliação empírica futura para validar sua configuração. Desta forma, acredita-se contribuir para a qualificação do cuidado de enfermagem culturalmente competente para a pessoa com deficiência.

\section{REFERÊNCIAS}

1. Chinn PL, Kramer MK. Integrated theory and knowledge development in nursing. 3. ed. Saint Louis: Elsevier/Mosby; 2011.

2. Geertz C. O saber local: novos ensaios em sociologia interpretativa. Rio de Janeiro: Vozes; 1997.

3. Andrews MM, Boyle JS, Editors. Transcultural concepts in nursing care. 5. ed. Philadelphia: Lippincott Williams \& Wilkins; 2005.

4. Shen Z. Cultural competence models in nursing: a selected annotated bibliography. J Transcult Nurs. 2004;15(4):317-22.

5. Brathwaite AEC. Evaluation of a cultural competence course. J Transcult Nurs 2005;16(4):361-9.

6. Ruthes RM, Cunha, ICKO. Entendendo as competências para aplicação na enfermagem. Rev Bras Enferm 2008;61(1):109-2.

7. Maia ER, Almeida SB, Oliveira WR, Pagliuca LMF. Care delivery to disabled people: competencies of community health agents. Rev Enferm UFPE On Line [periódico na internet]. 2009 out [acesso em 21 fev];3(4):151-9 Disponível em: <http://www.ufpe.br/revistaenfermagem/index. php/revista/article/viewArticle/104>

8. Instituto Brasileiro de Geografia e Estatística [homepage na internet]. Censo 2000. [acesso em 21 fev 2010]. Disponível em: <http://www.ibge.gov.br>

9. Organização Mundial da Saúde. Clasificación internacional de las deficiencias, actividades e participación: un manual de las dimensiones de la inhabilitación y su funcionamiento. Genebra: Organização Mundial de Saúde; 1997.

10. França ISX, Pagliuca LMF, Baptista RS, França EG, Coura AS, Souza JA. Violência simbólica no acesso das pessoas com deficiências às Unidades Básicas de Saúde. Rev Bras Enferm 2010;63(6):964-70.
11. Andrews MM, Boyle JS. Boyle transcultural concepts in nursing care. J Transcult Nurs 2002;13(3):178-80.

12. Campinha-Bacote, J. The process of cultural competence in the delivery of healthcare services: a model of care. J Transcult Nurs 2002;13(3):181-4.

13. Macêdo KNF, Pagliuca LMF, Almeida PC, Cardoso MV$\mathrm{LML}$, Rebouças CBA. Aspects of verbal communication between nurses and visually impaired people. Rev RENE 2009;10(2):29-36.

14. Wanderley LD, Barbosa GOL, Pagliuca LMF, Oliveira PMP, Almeida PC, Rebouças CBA. Comunicação verbal e não-verbal de mãe cega durante a higiene corporal da criança. Rev RENE 2010;11(nº esp):150-9.

15. Pagliuca LMF, Fiuza NLG, Rebouças CBA. Aspectos da comunicação da enfermeira com o deficiente auditivo. Rev Esc Enferm USP 2007;41(3):411-8.

16. França ISX, Pagliuca LMF. Social inclusion of disable people: achievements, challenges and implications for the nursing area. Rev Esc Enferm USP 2009;43(1):178-85.

17. Rebouças CBA, Cezario KG, Oliveira PMP, Pagliuca LMF. Pessoa com deficiência física e sensorial: percepção de alunos da graduação em enfermagem. Acta Paul Enferm 2011;24(1):80-6.

18. França ISX, Pagliuca LMF, Baptista RS. Política de inclusão do portador de deficiência: possibilidades e limites. Acta Paul Enferm 2008;21(1):112-6.

19. Vale EG, Pagliuca LMF. Construção de um conceito de cuidado de enfermagem: contribuição para o ensino de graduação. Rev Bras Enferm 2011;64(1):106-13.

20. Bezerra CP, Pagliuca LMF, Galvão MTG. Modelo de enfermagem baseado nas atividades de vida diária: adolescente diabética e deficiente visual. Rev Esc Anna Nery 2009;13(4):842-8. 